\title{
Molecular identification of endophytic fungi from torch ginger (Etlingera elatior) antagonist to phytopathogenic fungi
}

\author{
ANISA LUTFIA, ERMAN MUNIR ${ }^{\boldsymbol{*}}$, YURNALIZA YURNALIZA \\ Department of Biology, Faculty of Mathematics and Natural Sciences, Universitas Sumatera Utara, Jl. Bioteknologi No. 1, Medan 20155, North Sumatra, \\ Indonesia. Tel./fax.: +62-61-8214290, `email: erman@usu.ac.id
}

Manuscript received: 19 February 2020. Revision accepted: 21 May 2020.

\begin{abstract}
Lutfia A, Munir E, Yurnaliza. 2020. Molecular identification of endophytic fungi from torch ginger (Etlingera elatior) antagonist to phytopathogenic fungi. Biodiversitas 21: 2681-2689. Medicinal plants are valuable sources in exploration of endophytic microorganisms. The utilization of culturable endophytes, particularly endophytic fungi in pharmacological aspects has been reported in many literature. Zingiberaceae, being known as one of medicinal plant families, is commonly used in tropical countries, e.g Indonesia to treat health disorders and ailments. However, little information has been reported upon their associative endophytic fungi that may be used as biocontrol agents against phytopathogenic fungi. Efforts to elaborate on the symbiotic relationship between endophytic fungi and host to explain the antifungal ability from endophytes are presented in this study. Six fungal morphotypes were recovered from the rhizome fragment of torch ginger (Etlingera elatior (Jack) RM Smith) sampled from Sibayak forest, North Sumatra, Indonesia. All isolates displayed potential antifungal activities against Fusarium oxysporum, Ganoderma boninense, and Rigidoporus lignosus as representative phytopathogens in a dual culture plate assay. All fungal morphotypes were identified based on their 18S rDNA-ITS regions. Molecular identification revealed that most isolates were related to Trichoderma spp. while one isolate was identified as Pestaliotopsis sp. The endophytic assemblage of mostly Trichoderma strains in our study may reveal a mutual relationship of these soil fungi as root colonizer displaying biocontrol properties against phytopathogenic fungi to host.
\end{abstract}

Keywords: Endophytic fungi, Etlingera elatior, rDNA-ITS, Pestalotiopsis, Trichoderma

\section{INTRODUCTION}

Endophytic fungi are fungal community residing interor intracellular part of a plant exhibiting no apparent disease sign to a healthy plant. Recently, this form of interaction has brought an alternative way of discovering novel metabolites as the identification of plant secondary metabolites has been conducted progressively (Strobel and Daisy 2003; Kaul et al. 2012). The bioprospective study upon these microorganisms is based on the way that endophytic microorganisms may synthesize or even mimic the similar compound produced by their host plants hence increasing the probability of finding new compounds (Venieraki et al. 2017).

The rationale upon isolating potential endophytes is by choosing a plant with a historical background in traditional or ethnobotanical usage as practical medicine (Strobel 2003). Zingiberaceae is known as a multi-purpose plant family that already being utilized as spices and medicines in several countries of Southeast Asia. In Indonesia, Zingiberaceae has been reported as economical and medicinal plants in most ethnobotanical studies (Fernando et al. 2008; Auliani et al. 2014; Hartanto et al. 2014).

Torch ginger or kecombrang (Etlingera elatior (Jack) R.M. Smith) is a species in Zingiberaceae, widely cultivated and traditionally used as medicinal plants in Indonesia (Siregar and Pasaribu 2008). In North Sumatra, the species is commonly utilized as an ingredient in ethnobotanical medicine to treat cough and fever while also being added as spice or cekala by the Batak Karo subethnic, in traditional cuisine of arsik and terites (Silalahi et al. 2015; Silalahi and Nisyawati 2018). The ginger species is also well-studied for its biological properties such as anti-microbial, anti-oxidant, anti-tumor, antihyperglycemic, anti-hyperuricemic, anti-inflammatory, anti-larvae, anti-aging and functional properties as a topical medicine and containing phytochemicals, i.e. flavonoids, glycosides, phenols, saponins, steroids, tannins, and terpenoids (Juwita et al. 2018). Although the species has been comprehensively reported for its pharmacological properties, information regarding bioprospective study of their endophytic microorganisms is still limited.

Preliminary investigations have revealed the occurrence of endophytic fungi isolated from some unreported Zingiberaceous species in North Sumatra, such as Amomum centrocephalum, Etlingera littoralis, Hedychium coronarium, and Globba pendula, with major functional roles as biocontrol agents to pathogenic bacteria and phytopathogenic fungi (Lutfia et al. 2019a; Lutfia et al. 2019b; Lutfia et al. 2019c; Lutfia et al. 2019d). Based on the reports, we assume that endophytic fungi of torch ginger may also be effective as antagonistic agents, especially against phytopathogenic fungi. This research is then aimed to evaluate the antagonistic properties of culturable endophytic fungi isolated from the rhizome of $E$. elatior medicinal plants and to assign their identity based on molecular identification. 


\section{MATERIALS AND METHODS}

\section{Plant materials}

Plant sample, Etlingera elatior (Jack.) R. M. Smith was collected during exploration in the Sibayak forest, located in Deli Serdang District, North Sumatra. Sampling was conducted purposively without considering any climate and spatial factors. Plants anchoring to soils were dug up and cut to separate its shoots and roots. The root parts or rhizome were wrapped with paper and stored in plastic bags. Duplicate samples were collected separately to be identified at Herbarium Medanese, Universitas Sumatera Utara. In the laboratory, rhizomes were later cut into smaller pieces, and composites were made by pooling pieces into one bulk sample for each species of Zingiberaceae. The samples were then used in fungal isolation step.

\section{Isolation of endophytic fungi}

Step in isolating fungal endophytes was based on a previous report (Yurnaliza et al. 2014). Bulk samples from each species of Zingiberaceae were cleansed with mineral water to remove the remaining soil and dirt. The sample's surface was sterilized by dipping the samples into following sterilizing agents: $75 \% \mathrm{EtOH}(2 \mathrm{~min}), 5.3 \%$ $\mathrm{NaOCl}(5 \mathrm{~min}), 75 \% \mathrm{EtOH}(30 \mathrm{~s})$. The pieces were again washed several times with sterile deionized water to remove remaining solutions. Samples were blotted using Whatman filter paper and let to dry following cut into 1-2 minor pieces. The pieces were inoculated on Potato Dextrose Agar (Oxoid ${ }^{\mathrm{TM}}$ ) as isolation medium pre-added with standard antibiotic, chloramphenicol. Plates were incubated in ambient conditions for 3 days. Any visible fungal growth from each piece was then sub-cultured onto a new medium to preserve the strains. Each fungal strain was differentiated from their colony appearances.

\section{Antagonistic assay against phytopathogenic fungi}

Phytopathogenic fungal species used in this study were: Fusarium oxysporum, Ganoderma boninense, and Rigidoporus lignosus, collections of Laboratory of Microbiology, Department of Biology, Universitas Sumatera Utara, Medan, Indonesia. Phytopathogens were first grown in the PDA medium to obtain fresh mycelial plugs. Mycelial plugs of phytopathogens were then planted three days in advance at the center of the new PDA medium, followed by agar plugs of endophytic fungi in antagonism assay. Plates were incubated for 7 days at room temperature. The assay was conducted in triplicates. Colony Growth Inhibition (CGI) of phytopathogenic fungi was then calculated (Bivi et al. 2010):

$$
C G I(\%)=\frac{\left(R_{1}-R_{2}\right)}{R_{1}} \times 100
$$

Where, CGI is percentage (\%) of colony growth inhibition. $R_{I}$ represents the diameter of phytopathogens colony growth in the absence of fungal antagonist, $R_{2}$ represents the diameter of phytopathogens from antagonist direction. Each CGI results represented four arbitary levels of antagonism: Very Strong (++++) CGI $>75 \%$, Strong $(+++) 75 \geq$ CGI $>50 \%$, Mild (++) $50 \geq$ CGI $>25 \mathrm{~mm}$, Weak (+) $25 \geq$ CGI $>0 \%$, and None (-) CGI $=0 \%$.

\section{Methanolic extraction of dried rhizome}

The extraction procedure performed in this experiment was maceration. Ten grams of the shade-dried rhizome of Etlingera elatior were placed into a $250-\mathrm{mL}$ flask prior addition of $100 \mathrm{~mL}(1: 10, \mathrm{w} / \mathrm{v})$ of $\mathrm{MeOH}$. The flask was shaken at $120 \mathrm{rpm}$ for 5 days. After agitation, macerates were filtered through muslin cloth to separate extracts and debris. The extracts were then filtered (Whatman No.1) and further concentrated in a rotary evaporator under low pressure at $45^{\circ} \mathrm{C}$. The crude extracts in the form of paste were diluted in a sufficient amount of Dimethyl Sulfoxide (DMSO) $(\mathrm{w} / \mathrm{v})$. Antimicrobial assays against pathogenic bacteria and phytopathogenic fungi using $\mathrm{MeOH}$ extract were similar as previously performed by replacing the use of agar plugs to sterile blank discs (Oxoid ${ }^{\mathrm{TM}}$ ). The antimicrobial activities were categorized based as previously described.

\section{Fungal genomic DNA extraction}

Fungal genomic DNA extraction was performed following the technical procedure provided by Wizard ${ }^{\circledR}$ Genomic DNA Purification Kit Protocol (United States). $0.5 \mathrm{~g}$ of fresh mycelium from each isolate was picked and dissolved into a mixture of $600 \mu \mathrm{L}$ SDS Tris- $\mathrm{HCl} \mathrm{pH}$ 8.0, and $600 \mu \mathrm{L}$ phenol-chloroform. Homogenates were centrifuged at $4^{\circ} \mathrm{C}, 10000 \times \mathrm{g}$ for $45 \mathrm{~min}$. Pellets were further dissolved in $100 \mu \mathrm{L}$ Tris-EDTA. The DNA quality was assessed qualitatively $\left(\mathrm{A}_{260 / 280}, \mathrm{~A}_{260 / 230}\right)$.

\section{PCR amplification}

The rDNA-ITS region was amplified using the universal primer for fungi: ITS1 (5'CTTGGTCATTTAGAGGAAGTAA-3) and ITS4 (5'TCCTCCGCTTATTGATATGC-3') (Manter and Vivanco $2007)$ with reaction mixture composed of: $12 \mu \mathrm{L}$ nucleasefree water (NFW), $20 \mu \mathrm{L}$ GoTaq DNA Polymerase solution, $2 \mu \mathrm{L}$ ITS-1F primer solution, $2 \mu \mathrm{L}$ ITS-4 primer solution, $4 \mu \mathrm{L}$ DNA template solution with total volume of $40 \mu \mathrm{L}$ in Eppendorf tube. The PCR was conditioned as: 95 ${ }^{\circ} \mathrm{C}$ for $3 \mathrm{~min}$, followed by 35 cycles of $95{ }^{\circ} \mathrm{C}$ for $45 \mathrm{sec}, 55$ ${ }^{\circ} \mathrm{C}$ for $45 \mathrm{sec}, 72{ }^{\circ} \mathrm{C}$ for $45 \mathrm{sec}$, and final extension at $72{ }^{\circ} \mathrm{C}$ for $7 \mathrm{~min}$.

\section{Electrophoresis and DNA sequencing}

The result of DNA extraction or PCR products were visually checked on $1 \%$ agarose gels stained with Ethidium Bromide (EtBr) The gel was processed by immersing in a solution of Tris Acetate EDTA (TAE) buffer $1 \times$ following the run of device at $110 \mathrm{~V}$. After electrophoresis, the gel was examined under UV illumination inside a gel-doc $u v$ transilluminator. The PCR products were sequenced at Macrogen, Inc (Singapore). The acquired ITS sequences were submitted to NCBI Genbank database to obtain the accession numbers: MT053420, MT053421, MT053422, MT053423, MT053424, and MT053425. 


\section{Bioinformatics study}

Sequence pools were qualitatively assembled and trimmed based on the quality of electropherograms provided by the service agent using BioEdit ver. 7.2 (Hall 1999). The sequences were subjected to BLASTn search in National Center for Biotechnology Information (NCBI) database as compare with other fungal ITS sequences retrieved from database (Altschul et al 1990; Altschul et al 1997). Additional sequence identification was performed in TrichOKEY ver. 2.0 by the International Subcommission on Trichoderma and Hypocrea Taxonomy (ISTH) to confirm the presence of unique multiple loci in Trichoderma species (Druzhinina et al. 2005; Bissett et al. 2015). Multiple sequence alignment was performed to trimmed sequences using MUSCLE feature provided in MEGA ver. 7.0 (Edgar 2004; Kumar et al 2016). Phylogenetic tree of rDNA-ITS sequence was constructed based on the maximum likelihood fits of nucleotide substitution models which produced the lowest Bayesian Information Criterion (BIC) in order to select the appropriate statistical model, along with 1000x bootstrapping for reliability test of constructed trees (Felsenstein 1985; Nei and Kumar 2000).

\section{RESULTS AND DISCUSSION}

\section{Antagonistic endophytic fungi from Etlingera elatior}

Seven isolates of endophytic fungi were successfully recovered from rhizome fragments of E. elatior. The result of antagonism assay between isolated fungi and phytopathogenic fungi is presented in Table 1. A survey on Zingiberaceaen species has been reported in which 9 species were found inhabiting the Sibayak forest. The species were E. elatior, E. fimbriobracteata, E. punicea, and 6 unknown species of Etlingera (Siregar and Pasaribu 2008). Sibayak forest is then considered as a representative natural habitat for a diversity of Zingiberaceae species.

It can be seen that most isolates produced antifungal activities, CGI $>50 \%$ against three phytopathogenic fungi. Only one isolate, Ee03 did not show antifungal activity against $F$. oxysporum and less potential against the other two phytopathogenic fungi. Meanwhile, the methanolic extract of E. elatior rhizome was only able to display antifungal activities, CGI $<50 \%$ (Figure 1). Hence, five isolates excluding Ee03 were subjected to molecular identification using the ITS-rDNA region.

Mechanisms of antifungal activities from E. elatior phytochemical compounds and their derivatives have been suspected through interaction with the cell wall, cell membrane, and intracellular enzymatic inhibition (Ma et al. 2010; Sung and Lee 2010; Cheah et al. 2014). Floral extract of E. elatior was able to inhibit the colony growth of Candida albicans and Aspergillus niger (Lachumy et al. 2010). Furthermore, the floral extract of E. elatior was tested against anthracnose pathogen, Colletotrichum gloeosporioides which displayed potential antifungal activity towards the phytopathogen (Punnawich et al. 2009). Currently, only one report on endophytic bacterial isolates from $E$. elatior which displayed antifungal activities through assessment of their antagonistic interactions and antifungal crude metabolites. Solid-state fermentation of each bacterial culture was subjected to maceration within organic solvents. The difference in antifungal activities was observed in two results: hexane extract of one isolate, IAK3 was more effective against $F$. oxysporum and $S$. rolfsii while ethyl acetate extract of IAK9 was more effective against $R$. solani, $R$. microsporus, Curvularia, and Saprolegnia (Suryanto et al. 2016).

Table 1. Antagonistic properties by endophytic fungi isolated from Etlingera elatior

\begin{tabular}{lccc}
\hline Isolate & \multicolumn{3}{c}{ Colony growth inhibition } \\
& $\begin{array}{c}\text { Fusarium } \\
\text { oxysporum }\end{array}$ & $\begin{array}{c}\text { Ganoderma } \\
\text { boninense }\end{array}$ & $\begin{array}{c}\text { Rigidoporus } \\
\text { lignosus }\end{array}$ \\
\hline $\mathrm{Ee} 01$ & +++ & +++ & +++ \\
$\mathrm{Ee} 02$ & +++ & ++++ & +++ \\
$\mathrm{Ee} 03$ & - & ++ & + \\
$\mathrm{Ee} 04$ & +++ & +++ & +++ \\
$\mathrm{Ee} 05$ & +++ & ++++ & +++ \\
$\mathrm{Ee} 06$ & +++ & +++ & +++ \\
$\mathrm{Ee} 07$ & +++ & +++ & +++ \\
$\mathrm{MeOH}$ & ++ & ++ & ++ \\
\hline
\end{tabular}

Note: Antagonism levels based on Colony Growth Inhibition (\%): Very Strong $(++++)$ CGI $>75 \%$, Strong $(+++) 75 \geq$ CGI $>50 \%$, Mild $(++) 50 \geq$ CGI $>25 \mathrm{~mm}$, Weak $(+) 25 \geq$ CGI $>0 \%$, and None (-) CGI $=0 \%$
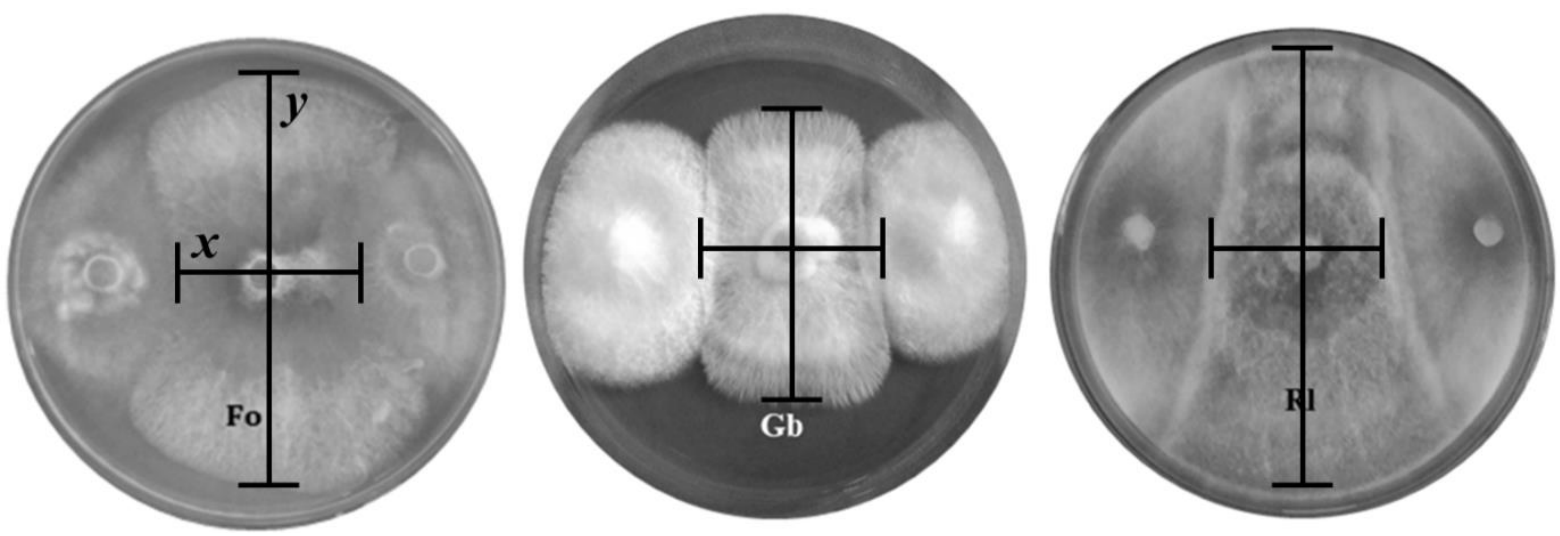

Figure 1. The inhibitory activities by endophytic fungi against phytopathogenic fungi (center) as revealed from its horizontal $(x)$ apparent to its vertical colony growth (y). Fo: Fusarium oxysporum; Gb: Ganoderma boninense; Rl: Rigidoporus lignosus 
While other reports have revealed the potency of endophytic fungi as antifungal agents from other species of Zingiberaceae, little is known from E. elatior. Varied inhibitory activities were produced by endophytic fungi of common ginger (Zingiber officinale). Three isolates identified as Curvularia affinis, Fusarium solani, and Glomerella cingulata were able to produce high antagonistic activity with CGI $>65 \%$ against $F$. oxysporum in dual culture assay (Ginting et al. 2013). In addition, studies on testing endophytic fungi against phytopathogenic fungi such as $G$. boninense and $R$. lignosus are still limited.

\section{Electrophoresis image of rDNA-ITS amplicons}

DNA extracts from all endophytic fungal isolates were obtained from 5-days old mycelium. The active-growing mycelia were sampled and crushed in the absence of spores which may affect the extraction process. The presence of a single band from each well indicated the results of PCR amplification using ITS1 and ITS4 primers (Figure 2). A 1 kbp (Promega Corp., USA) was used as molecular marker in this study.

\section{Endophytic fungi identification based on BLASTn}

Sequence analysis of six isolates was done to reveal the identity of endophytic fungi to the genus/species level. Oligonucleotide fragment of ITS-rDNA sequences was compared with their reference sequences. Molecular identification of selected five endophytic fungi revealed that 5 of them were relatively closed to-Trichoderma (Tables 2, 4-7), while Ee02 was identified as Pestalotiopsis, each of them was aligned with the ITSrDNA sequence in GenBank database (Table 3). Based on nucleotide database pairing in GeneBank, there are numerous suggestions on identification results as indicated by the list of species ranked by their scores, query covers, and maximum identities. In this study, however, majority of results showed no distinction in overall scores although the identification was placed in the same genus. Here we report the 10 species listed from the BLASTn results. Isolate Ee01 was closely related to Trichoderma sp. originating from Colombia, followed by the other species i.e. T. neotropicale and T. harzianum and Hypocrea sp., within the score of 1086 and similarities of $100 \%$. In short, isolate Ee02 and Ee05 were identified as Pseudopestalotiopsis theae $(100 \%)$ and T. caribbaeum $(100 \%)$, respectively, while isolate $\mathrm{Ee} 04, \mathrm{Ee} 06$, and $\mathrm{Ee} 07$ were similarly designated as an unidentified species of Trichoderma sp.

\section{Trichoderma identification based on TrichOKEY}

Molecular identification of these endophytic fungi, especially in the genus Trichoderma, may be explored thoroughly by using the online-based identification or TrichOKEY (http://www.isth.info/tools/molkey/). The diagnostic was promoted due to the findings on unique oligonucleotide barcodes (hallmarks) residing within the internal transcribed spacer 1 and 2 (ITS1 and 2)
(Druzhinina et al. 2005). The genus-specific hallmarks (GSH) from each sequence were found to be present in different nucleotide sites (anchors) as presented in Table 7. Based on this identification, isolate $\mathrm{Ee} 01$ was designated as Hypocrea lixii or T. harzianum in section I of LixiiCaptotron clade, while three remaining sequences namely $\mathrm{Ee} 04, \mathrm{Ee} 05$, and $\mathrm{Ee} 07$ were designated as T. koningiopsis in section XII of Rufa clade. Only isolate Ee06 which was designated as unidentified Trichoderma in the section of the similar XII of Rufa clade. In addition to TrichOKEY, is the web-based TrichoBLAST, by integrating the GenBank rDNA-ITS or multilocus database which have also been helping in the Trichoderma-related study (Kopchinsky et al. 2005). Although the sequence database (TrichOKEY) has been upgraded to additional Trichoderma species, there are still differences in identified species which must be confirmed through evolutionary analysis (Bissett et al. 2015). Phylogenetic trees were then constructed to discriminatively assign the identities to all endophytic fungal isolates.

\section{Phylogenetic analysis}

The maximum likelihood statistical method and Kimura-2 parameter model were used to construct the phylogenetic trees of the endophytic fungal isolates from Etlingera elatior. Here, we constructed two phylogenetic trees to divide between Trichoderma and Pestalotiopsis members (Figures 3 and 4). Based on the evolutionary distance and bootstrap value (BV) $\geq 60 \%$, each isolate was reliably assigned to the species level of identification. Therefore, the isolate Ee01 was identified as Trichoderma neotropicale with an accession code: MT053420; isolate Ee02 as Pestalotiopsis sp (MT053421); the isolates Ee04 and Ee07, both identified as T. koningiopsis (MT053422, MT053425); isolate Ee05 as T. caribbaeum (MT053423); and isolate Ee06 as T. gamsii (MT053424).

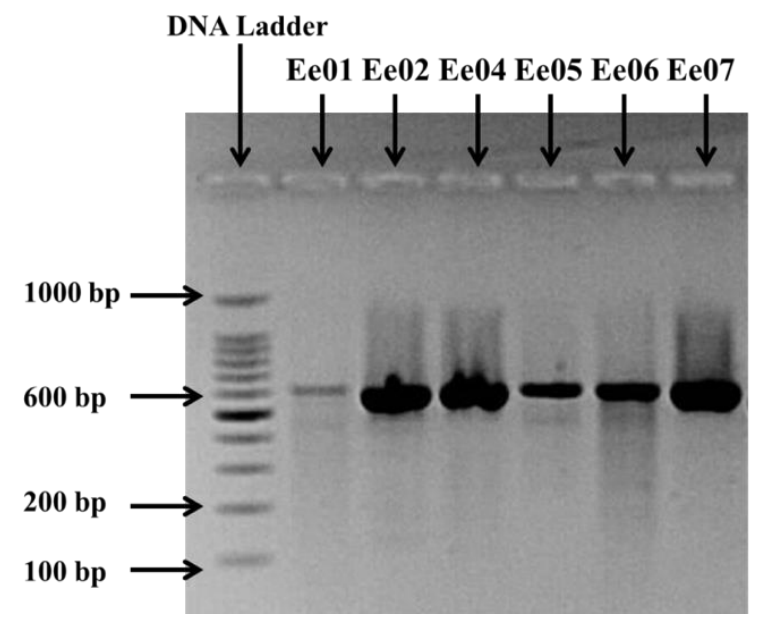

Figure 2. Electrophoresis product of ITS-rDNA amplicons from antagonistic fungal isolates with product size $\pm 600 \mathrm{bp}$. 
Table 2. BLASTn result of isolate Ee01 identification

\begin{tabular}{|c|c|c|c|c|c|c|}
\hline Species & Isolate/strain & Accession code & Score & $\begin{array}{l}\text { Query } \\
\text { cover }\end{array}$ & $\begin{array}{c}\text { Max } \\
\text { identity }\end{array}$ & Source \\
\hline Trichoderma sp. & GH40 & MN602871.1 & 1086 & $100 \%$ & $100.00 \%$ & Colombia \\
\hline Trichoderma netropicale & CBS 130633 & MH865818.1 & 1086 & $100 \%$ & $100.00 \%$ & Peru $^{\mathrm{a}}$ \\
\hline Trichoderma harzianum & $42315 \mathrm{bDRJ}$ & MF782822.1 & 1086 & $100 \%$ & $100.00 \%$ & Poland \\
\hline Trichoderma sp. & S259 & MF076625.1 & 1086 & $100 \%$ & $100.00 \%$ & Brazil \\
\hline Trichoderma harzianum & Th.12 & KJ010951.1 & 1086 & $100 \%$ & $100.00 \%$ & Algeria \\
\hline Trichoderma sp. & AM140-P1T5R & KT264464.1 & 1086 & $100 \%$ & $100.00 \%$ & USA \\
\hline Hypocrea sp. & AE-2013 & KF746146.1 & 1086 & $100 \%$ & $100.00 \%$ & Panama ${ }^{b}$ \\
\hline Hypocrea sp. & AE-2013 & KF746100.1 & 1086 & $100 \%$ & $100.00 \%$ & Panama $^{\mathrm{b}}$ \\
\hline Trichoderma harzianum & RSPG_28 & KC478545.1 & 1086 & $100 \%$ & $100.00 \%$ & Thailand \\
\hline Trichoderma harzianum & CKP01 & KC330218.1 & 1086 & $100 \%$ & $100.00 \%$ & India \\
\hline
\end{tabular}

Note: ${ }^{\mathrm{a}} \mathrm{Vu}$ et al (2019), ${ }^{\mathrm{b}}$ Higginbotham et al (2014)

Table 3. BLASTn result of isolate Ee02 identification

\begin{tabular}{lllcccc}
\hline Species & Isolate/strain & $\begin{array}{l}\text { Accession } \\
\text { code }\end{array}$ & Score & $\begin{array}{l}\text { Query } \\
\text { cover }\end{array}$ & $\begin{array}{c}\text { Max } \\
\text { identity }\end{array}$ & Source \\
\hline Pseudopestalotiopsis theae & AXLKY2019015 & MN202256.1 & 1090 & $100.00 \%$ & $100.00 \%$ & China \\
Pestalotiopsis sp. & TAP18N011 & LC427147.1 & 1090 & $100.00 \%$ & $100.00 \%$ & Japan \\
Pestalotiopsis kenyana & BM4 & MK910059.1 & 1090 & $100.00 \%$ & $100.00 \%$ & China \\
Pestalotiopsis kenyana & PC4 & MK910055.1 & 1090 & $100.00 \%$ & $100.00 \%$ & China \\
Pestalotiopsis sp. & CQ1A2 & KU252298.1 & 1090 & $100.00 \%$ & $100.00 \%$ & China \\
Pestalotiopsis sp. & CQ1A1 & KU252297.1 & 1090 & $100.00 \%$ & $100.00 \%$ & China \\
Pestalotiopsis sp. & HUN1A4 & KU252296.1 & 1090 & $100.00 \%$ & $100.00 \%$ & China \\
Pestalotiopsis sp. & ZJ2A7 & KU252295.1 & 1090 & $100.00 \%$ & $100.00 \%$ & China \\
Pestalotiopsis sp. & HB2A5 & KU252294.1 & 1090 & $100.00 \%$ & $100.00 \%$ & China \\
Pestalotiopsis sp. & HB2A2 & KU252291.1 & 1090 & $100.00 \%$ & $100.00 \%$ & China \\
\hline
\end{tabular}

Table 4. BLASTn result of isolate Ee04 identification

\begin{tabular}{lllcccc}
\hline Species & Isolate/strain & $\begin{array}{l}\text { Accession } \\
\text { code }\end{array}$ & Score & $\begin{array}{l}\text { Query } \\
\text { cover }\end{array}$ & $\begin{array}{c}\text { Max } \\
\text { identity }\end{array}$ & Source \\
\hline Trichoderma sp. & SDAS203143 & MK870992.1 & 1094 & $100 \%$ & $100.00 \%$ & China \\
Trichoderma sp. & HZA8 & MH624143.1 & 1094 & $100 \%$ & $100.00 \%$ & China \\
Trichoderma sp. & HZA5 & MH624140.1 & 1094 & $100 \%$ & $100.00 \%$ & China \\
Trichoderma dorotheae & CBS 119089 & MH863050.1 & 1094 & $100 \%$ & $100.00 \%$ & New Zealand \\
Trichoderma koningiopsis & CRM-RCH169 & MK396898.1 & 1094 & $100 \%$ & $100.00 \%$ & Mexico \\
Trichoderma sp. & CM-CNRG444 & MK311190.1 & 1094 & $100 \%$ & $100.00 \%$ & Mexico \\
Trichoderma sp. & yi1481_1 & MH284844.1 & 1094 & $100 \%$ & $100.00 \%$ & China \\
Trichoderma sp. & yi1440_1 & MH284799.1 & 1094 & $100 \%$ & $100.00 \%$ & China \\
Trichoderma sp. & Cg05 & MF101380.1 & 1094 & $100 \%$ & $100.00 \%$ & South Korea \\
Trichoderma koningiopsis & UFSMQ40 & KX496883.1 & 1094 & $100 \%$ & $100.00 \%$ & Brazil \\
\hline
\end{tabular}

Note: ${ }^{a}$ Vu et al (2019)

Table 4. BLASTn result of isolate Ee05 identification

\begin{tabular}{|c|c|c|c|c|c|c|}
\hline Species & Isolate/strain & $\begin{array}{l}\text { Accession } \\
\text { code }\end{array}$ & Score & $\begin{array}{l}\text { Query } \\
\text { cover }\end{array}$ & $\begin{array}{c}\text { Max } \\
\text { identity }\end{array}$ & Source \\
\hline Trichoderma caribbaeum & CBS 119093 & MH863051.1 & 1335 & $100 \%$ & $100.00 \%$ & Guadeloupe $^{\mathrm{a}}$ \\
\hline Trichoderma sp. & 16 BRO-2013 & KF367557.1 & 1322 & $100 \%$ & $99.59 \%$ & Portugal $^{\mathrm{b}}$ \\
\hline Trichoderma sp. & 15 BRO-2013 & KF367555.1 & 1322 & $100 \%$ & $99.59 \%$ & Portugal $^{b}$ \\
\hline Hypocrea koningii & ATCC 64262 & AJ301990.1 & 1322 & $100 \%$ & $99.59 \%$ & Germany \\
\hline Trichoderma gamsii & Clone 2 & KM491887.1 & 1320 & $100 \%$ & $99.59 \%$ & Portugal \\
\hline Trichoderma gamsii & Clone 1 & KM491886.1 & 1318 & $99 \%$ & $99.59 \%$ & Portugal \\
\hline Trichoderma atroviride & NG_13 & HQ115671.1 & 1313 & $100 \%$ & $99.32 \%$ & Austria ${ }^{c}$ \\
\hline Trichoderma atroviride & - & EF417482.1 & 1307 & $100 \%$ & $99.19 \%$ & Iran \\
\hline Trichoderma koningii & APSAC 01 & KY886134.1 & 1303 & $100 \%$ & $99.19 \%$ & India \\
\hline Trichoderma koningii & S54 & MF076590.1 & 1287 & $98 \%$ & $99.18 \%$ & Brazil \\
\hline
\end{tabular}

Note: ${ }^{\mathrm{a}} \mathrm{Vu}$ et al (2019), ${ }^{\mathrm{b}}$ Oliveira et al (2013), ${ }^{\mathrm{c}}$ Gorfer et al (2011) 
Table 5. BLASTn result of isolate Ee06 identification

\begin{tabular}{|c|c|c|c|c|c|c|}
\hline Species & Isolate/strain & $\begin{array}{l}\text { Accession } \\
\text { code }\end{array}$ & Score & $\begin{array}{l}\text { Query } \\
\text { cover }\end{array}$ & $\begin{array}{c}\text { Max } \\
\text { identity }\end{array}$ & Source \\
\hline Trichoderma sp. & HSrS3-7-MR & MK793785.1 & 1094 & $100 \%$ & $100.00 \%$ & Mexico \\
\hline Trichoderma sp. & RS3-4-MA & MK793784.1 & 1094 & $100 \%$ & $100.00 \%$ & Mexico \\
\hline Trichoderma sp. & RS3-3-MA & MK793783.1 & 1094 & $100 \%$ & $100.00 \%$ & Mexico \\
\hline Trichoderma gamsii & TRS125 & KP009326.1 & 1094 & $100 \%$ & $100.00 \%$ & Poland \\
\hline Trichoderma gamsii & Clone 2 & KM491887.1 & 1094 & $100 \%$ & $100.00 \%$ & Portugal \\
\hline Trichoderma gamsii & Clone 1 & KM491886.1 & 1094 & $100 \%$ & $100.00 \%$ & Portugal \\
\hline Trichoderma gamsii & TJG4 & KJ652466.1 & 1094 & $100 \%$ & $100.00 \%$ & China \\
\hline Trichoderma gamsii & CQBN3005 & JQ040342.1 & 1094 & $100 \%$ & $100.00 \%$ & China \\
\hline Trichoderma gamsii & ICC080 & GQ351597.1 & 1094 & $100 \%$ & $100.00 \%$ & Spain \\
\hline Trichoderma gamsii & MIAE00029 & HM176559.1 & 1094 & $100 \%$ & $100.00 \%$ & France $^{\mathrm{a}}$ \\
\hline
\end{tabular}

Note: ${ }^{a}$ Anees et al (2016)

Table 6. BLASTn result of isolate Ee07 identification

\begin{tabular}{|c|c|c|c|c|c|c|}
\hline Species & Isolate/strain & $\begin{array}{c}\text { Accession } \\
\text { code }\end{array}$ & Score & $\begin{array}{l}\text { Query } \\
\text { cover }\end{array}$ & $\begin{array}{c}\text { Max } \\
\text { identity }\end{array}$ & Source \\
\hline Trichoderma sp. & SDAS203143 & MK870992.1 & 1094 & $100 \%$ & $100.00 \%$ & China $^{\mathrm{a}}$ \\
\hline Trichoderma sp. & AA-2019 & MH624143.1 & 1094 & $100 \%$ & $100.00 \%$ & China \\
\hline Trichoderma sp. & AA-2019 & MH624140.1 & 1094 & $100 \%$ & $100.00 \%$ & China \\
\hline Trichoderma dorotheae & CBS 119089 & МH863050.1 & 1094 & $100 \%$ & $100.00 \%$ & New Zealand ${ }^{b}$ \\
\hline Trichoderma koningiopsis & CRM-RCH169 & MK396898.1 & 1094 & $100 \%$ & $100.00 \%$ & Mexico \\
\hline Trichoderma sp. & CM-CNRG444 & MK311190.1 & 1094 & $100 \%$ & $100.00 \%$ & Mexico \\
\hline Trichoderma sp. & yi1481_1 & MH284844.1 & 1094 & $100 \%$ & $100.00 \%$ & China \\
\hline Trichoderma sp. & yi1440_1 & MH284799.1 & 1094 & $100 \%$ & $100.00 \%$ & China \\
\hline Trichoderma sp. & $\mathrm{Cg} 05$ & MF101380.1 & 1094 & $100 \%$ & $100.00 \%$ & South Korea \\
\hline Trichoderma koningiopsis & UFSMQ40 & KX496883.1 & 1094 & $100 \%$ & $100.00 \%$ & Brazil \\
\hline
\end{tabular}

Note: ${ }^{\mathrm{a}} \mathrm{Hu}$ et al (2019), ${ }^{\mathrm{b}} \mathrm{Vu}$ et al (2019)

Table 7. TrichOKEY result of Trichoderma isolates identification

\begin{tabular}{llccccc}
\hline \multirow{2}{*}{ Isolate } & \multirow{2}{*}{ Species } & \multicolumn{4}{c}{ Genus-Specific Hallmarks (GSH) position } \\
\cline { 2 - 7 } & Hypocrea lixiil & $\mathbf{1}^{\text {st }}$ Anchor & $\mathbf{2}^{\text {nd }}$ Anchor & $\mathbf{3}^{\text {rd }}$ Anchor & $\mathbf{4}^{\text {th }}$ Anchor & $\mathbf{5}^{\text {th }}$ Anchor \\
\hline \multirow{2}{*}{ Ee01 } & Trichoderma harzianum & 71 & 93 & 254 & 412 & 512 \\
Ee04 & Trichoderma koningiopsis & 86 & 107 & 253 & 411 & 513 \\
Ee05 & Trichoderma koningiopsis & 87 & 108 & 254 & 412 & 514 \\
Ee06 & Unidentified/Trichoderma sp. & 83 & 104 & 251 & 409 & 511 \\
Ee07 & Trichoderma koningiopsis & 86 & 107 & 253 & 411 & 513 \\
\hline
\end{tabular}

Isolation of Trichoderma isolates/strains from several medicinal plants has been reported from various plant host species. Trichoderma strains were known to colonize several parts of Tinospora cordifolia, Piper nigrum, and Hedychium coronarium, some representatives of medicinal plants used in Western Ghats, India. The isolated strains secreted extracellular enzymes, i.e. asparaginase and cellulase that might lead to industrial bioprospecting study in the future (Uzma et al. 2016). Trichoderma piluliferum isolated from a medicinal plant, Bauhinia forficata from Brazil was atse known to secrete other extracellular enzymes, i.e. xylanase and lipase (Jadson et al. 2015). Trichoderma koningiopsis isolated from Artemisia argyi from China was known to synthesize and secreted novel fungal polyketides in the culture extracts. The polyketide compounds exhibited antibacterial activity against human pathogen, Escherichia coli, and antifungal activities against agro-pathogenic fungi (Shi et al., 2017). Trichoderma atroviride PG87 isolated from Panax ginseng significantly reduced the disease incidence by the ginseng pathogenic fungi, the Botrytis cinerea, and Cylindrocarpon destructans, (Park et al. 2018).

A similar potential strain of Trichoderma was also obtained from soil samples of Andaman and Nicobar Islands, India. All isolates produced significant inhibitions (CGI $>50 \%$ ) to mycelium of Sclerotium rolfsii, a root and foliar phytopathogen in a dual culture plate assay (Kumar et al. 2012). The assemblage of dominantly Trichoderma in the rhizome of E. elatior was considered logical since the lifestyle of these fungi as common soil inhabitants with high population in nature. A comprehensive review has stated upon their use as a biological control agent in suppressing phytopathogenic fungi either in laboratory or field study. Members of Trichoderma are known as prominent Biological Control Agent (BCA) through several mechanisms: plant growth promotion, systemic-acquired resistances, fast colonization, antifungal production, and mycoparasitism (Mukesh et al. 2016). 


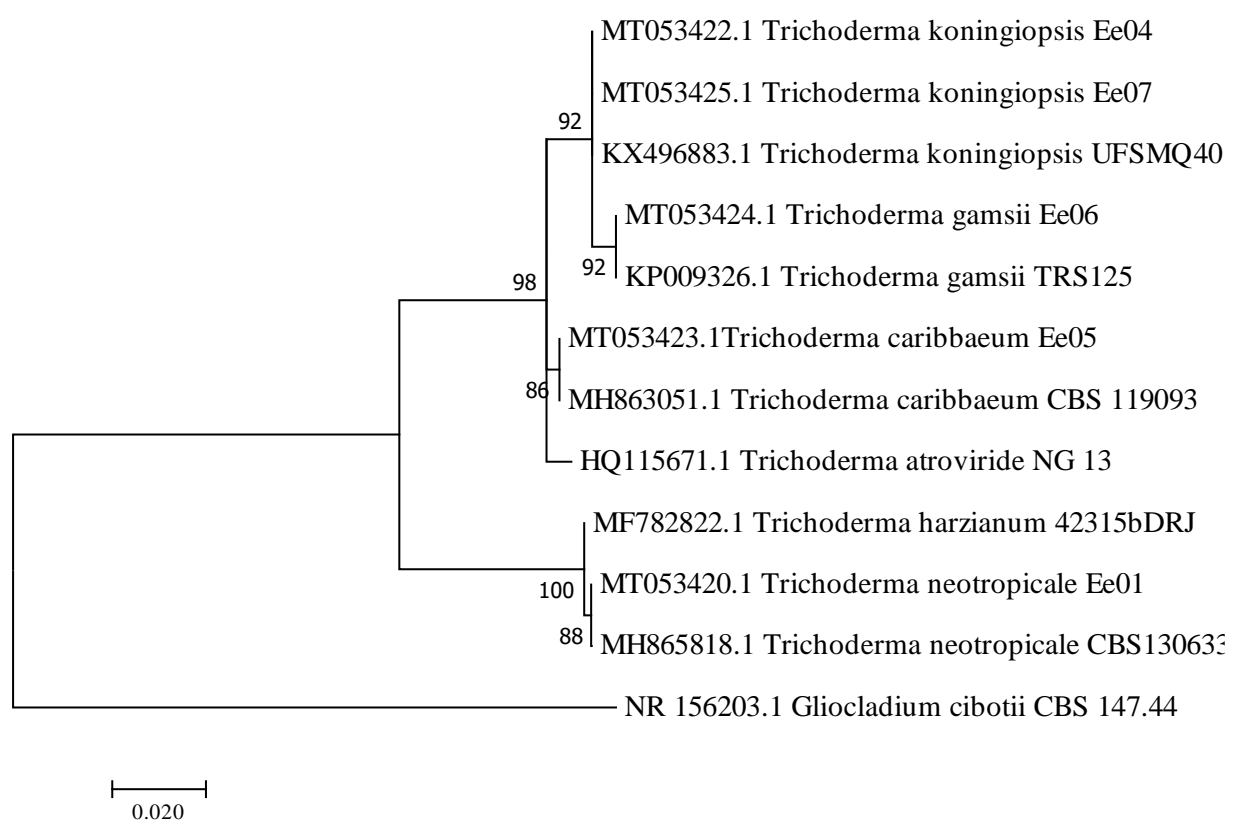

Figure 3. Phylogenetic tree of Trichoderma isolates and its members based on rDNA-ITS sequence similarity

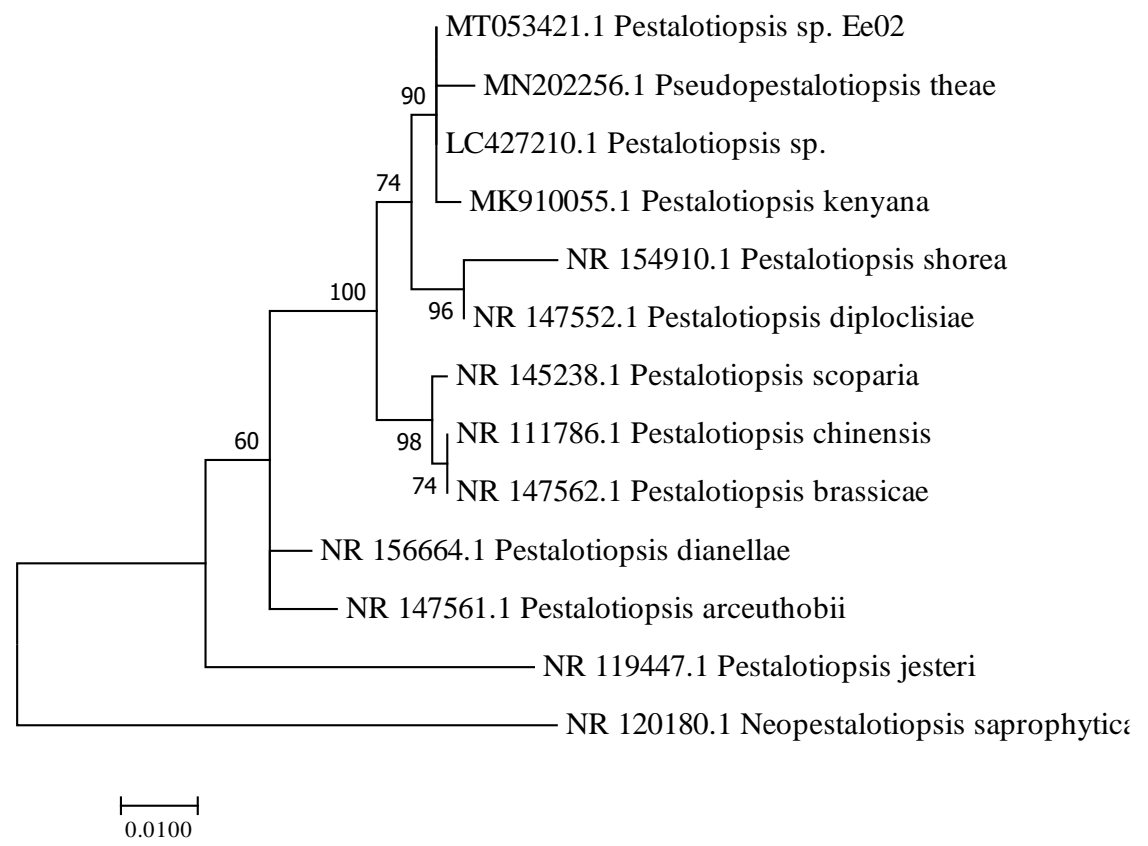

Figure 4. Phylogenetic tree of Pestalotiopsis isolate Ee02 and its based on rDNA-ITS sequence similarity

A study reported the laboratory test of Trichoderma sp. strain $\mathrm{T} 11$ isolated from the rhizosphere of Zingiber officinale. The mechanism proposed in explaining the mycelium inhibition of $F$. oxysporum is through the synthesis of chemo-attractants by Trichoderma followed by rapid colonization or mycoparasitism towards $F$. oxysporum colony (Sharma 2011). In addition, the dominantly isolated Trichoderma spp. were also reported from Elettaria sp. from North Sumatrar revealing their high population as soil mycoflora (Munir et al. 2019). Our finding on their colonization in E. elatior is a new report that may reveal further aspects in supporting the hardy nature of Zingiberaceaen species to exist in the disturbed habitat (Habsah et al. 2000).

The genus Pestalioptopsis exists as a dominant endophytic fungus in the world's rainforest with potential producer of a diverse chemical compounds, mostly known as antibacterial compounds (Deshmukh et al. 2014). The 
assemblage of endophytic Pestaliotopsis species from plants of Podocarpaceae, Theaceae, and Taxaceae was documented in Southern China. The study concluded that Pestaliotopsis species was not generally specific to host plants explaining their diversity in temporal and spatial scale (Wei et al. 2007).

Both genera, Pestaliotopsis and Trichoderma obtained from our study has been reported as potential BCA against phytopathogenic fungi. A field study has been conducted to assess the potential application of Pestaliotopsis and Trichoderma isolated from cacao and cupuac trees. The strains successfully colonized the axenic culture of cacao plantlets and produced a lesser diseased plants in comparison to a positive control (Hanada et al. 2010). A key feature in successful Trichoderma application as BCA is influenced by its greatest quantity or former presence in soils. Control of damping-off in tobacco plants caused by Rhizoctonia solani is reported the best by applying the highest inoculum $(\log 4 \mathrm{CFU} / \mathrm{g})$ of Trichoderma strains with sowing (Gveroska 2013). Hence, a field study in proving the effectiveness of our isolated strains as BCA is needed to assess their efficacies.

The relationship among specific strains with their hosts may be revealed through comprehensive studies. Exploration of specific interaction of Trichoderma species so far has been reported with Cupressaceae. The Trichoderma strains demonstrated significant bioactivities leading to novel agrochemical/drug discovery and as potential BCAs in the future (Hosseyni-Moghaddam and Soltani 2014). This part of our study will also further evaluate and reveal the maximum potency of the diverse endophytic fungi of Zingiberaceae as a potential biocontrol agent for the application in agriculture.

\section{ACKNOWLEDGEMENTS}

This research was funded by Universitas Sumatera Utara under TALENTA Grant for Professor Research Scheme (contract Number: 427/UN5.2.3.1/PPM/KPTALENTA USU/2018) and Ministry of Research Technology and Higher Education, Republic of Indonesia under the scheme of PMDSU Research Grant (contact number 152/SP2H/LH/DRPM/2018).

\section{REFERENCES}

Altschul SF, Gish W, Miller W, Myers EW, Lipman DJ. 1990. Basic local alignment search tool. J. Mol. Biol. 215: 403-410.

Altschul SF, Madden TL, Schäffer AA, Zhang J, Zhang Z, Miller W, Lipman DJ. 1997. Gapped BLAST and PSI-BLAST: a new generation of protein database search programs. Nucleic Acids Res 25: 3389-3402.

Anees M, Tronsmo A, Edel-Hermann V, Hjeljord LG, Heraud C, Steinberg C. 2010. Characterization of field isolates of Trichoderma antagonistic against Rhizoctonia solani. Fungal Biol 114 (9): 691701.

Auliani A, Fitmawati F, Sofiyanti N. 2014. Studi etnobotani famili Zingiberaceae dalam kehidupan masyarakat lokal di Kecamatan Siak Hulu Kabupaten Kampar. Jurnal Online Mahasiswa FMIPA 1 (2): 526-533. [Indonesian]
Bezerra JDP, Nascimento CCF, Barbosa RN, Silva DCV, Svedese VM, Silva-Nogueira EB, Gomes BS, Paiva LM, Souza-Motta CM. 2015. Endophytic fungi from medicinal plant Bauhinia forficata: diversity and biotechnological potential. Braz J Microbiol 57: 49-57.

Bissett J, Gams W, Jaklitsch W, Samuels GJ. 2015. Accepted Trichoderma names in the year 2015. IMA Fungus 6 (2): 263-295.

Bivi MR, Farhana MSN, Khairulmazmi A, Idris A. 2010. Control of Ganoderma boninense : a causal agent of basal stem rot disease in oil palm with endophyte bacteria in vitro. Intl J Agr Biol 12: 833-839.

Cheah, HL, Lim V, Sandai D. 2014. Inhibitors of the glyoxylate cycle enzyme ICL1 in Candida albicans for potential use as antifungal agents. PLoS ONE 9 (4): e95951. DOI: 10.1371/journal.pone.0095951.

Deshmukh SK, Verekar SA, Bhave SV. 2014. Endophytic fungi: a reservoir of antibacterials. Front Microbiol 5: 1-43.

Dzurhinina IS, Kopchinskiy AG, Komon M, Bissett J, Szakacs G, Kubicek CP. 2005. An oligonucleotide barcode for species identification in Trichoderma and Hypocrea. Fungal Gen Biol 42: 813-828.

Edgar RC. 2004. MUSCLE: Multiple sequence alignment with high accuracy and high throughput. Nucleic Acids Res 32 (5): 1792-1797.

Felsenstein J. 2009. Confidence limits on phylogenies : an approach using the bootstrap. Evolution 39 (4): 783-791.

Fernando O, Nursyahra, Rizki. 2008. Studi pemanfaatan tumbuhan familia Zingiberaceae yang berkhasiat obat di Kecamatan Luhak Nan Duo Kabupaten Pasaman Barat. Pendidikan Biologi 4 (2): 1-5. [Indonesian]

Ginting RCB, Sukarno N, Widyastuti U, Darusman LK, Kanaya S. 2013. Diversity of endophytic fungi from red ginger (Zingiber officinale Rosc.) plant and their inhibitory effect to Fusarium oxysporum plant pathogenic fungi. Hayati J Biosci 20 (3): 127-137.

Gorfer M, Blumhoff M, Klaubauf S, Urban A, Inselsbacher E, Bandian D, Mitter B, Sessitsch A, Wanek W, Strauss J. 2011. Community profiling and gene expression of fungal assimilatory nitrate reductases in agricultural soil. ISME J 5 (11): 1771-1783.

Gveroska B. 2013. Relationships of Trichoderma spp. quantity in soil to reducing the dampingoff in tobacco seedlings. Bulg J Agri Sci 19 (4): 666-674.

Habsah M, Amran M, Mackeen MM, Lajis NH, Kikuzaki H, Nakatani N, Rahman AA, Ghafar, Ali AM. 2000. Screening of Zingiberaceae extracts for antimicrobial and antioxidant activities. J Ethnopharmacol 72 (3): 403-410.

Hall TA. 1999. BioEdit: a user-friendly biological sequence alignment editor and analysis program for Windows 95/98/NT. Nucleic Acids Symp Ser 41: 95-98.

Hanada RE, Pomella AWV, Costa HS, Bezerra JZ, Loguercio LL, Pereira JO. 2010. Endophytic fungal diversity in Theobroma cacao (Cacao) and T. grandiflorum (Cupuaçu) trees and their potential for growth promotion and biocontrol of black-pod disease. Fungal Biol 114 (1112): $901-910$.

Hartanto S, Fitmawati, Sofiyanti N. 2014. Studi etnobotani famili Zingiberaceae dalam kehidupan masyarakat lokal di Kecamatan Pangean Kabupaten Kuantan Singingi, Riau. Biosaintifika 6 (2): 98108. [Indonesian]

Higginbotham S, Wong WR, Linington RG, Spadafora C, Iturrado L, Arnold AE. 2014. Sloth hair as a novel source of fungi with potent anti-parasitic, anti-cancer and anti-bacterial bioactivity. PLoS ONE 9 (1): e84549. DOI: 10.1371/journal.pone.0084549.

Hosseyni-Moghaddam MS, Soltani J. 2014. Bioactivity of endophytic Trichoderma fungal species from the plant family Cupressaceae. Ann Microbiol 64 (2): 753-761.

Hu J, Zhou Y, Chen K, Li J, Wei Y, Wang Y, Wu Y, Ryder MH, Yang H, Denton MD. 2019. Large-scale Trichoderma diversity was associated with ecosystem, climate and geographic location. Environ Microbiol. DOI: $10.1111 / 1462-2920.1479$.

Juwita T, Puspitasari IM, Levita J. 2018. Torch ginger (Etlingera elatior): a review on its botanical aspects, phytoconstituents and pharmacological activities. Pak J Biol Sci 21 (4): 151-165.

Kaul S, Gupta S, Ahmed M, Dhar MK. 2012. Endophytic fungi from medicinal plants: a treasure hunt for bioactive metabolites. Phytochem Rev 11 (4): 487-505.

Kimura M. 1980. A simple method for estimating evolutionary rate of base substitutions through comparative studies of nucleotide sequences. J Mol Evol 16: 111-120. 
Kopchinskiy, A., Komoń, M. Kubicek, C. P. \& Druzhinina, I. S. (2005. TrichoBLAST: a multilocus database for Trichoderma and Hypocrea identifications. Mycol Res 109 (6): 658-660.

Kumar K, Amaresan N, Bhagat S, Madhuri K, Srivastava RC. 2012. Isolation and characterization of Trichoderma spp. for antagonistic activity against root rot and foliar pathogens. Indian J Microbiol 52 (2): 137-144

Lachumy SJT, Sasidharan S, Sumathy S, Zakaria Z. 2010. Pharmacological activity, phytochemical analysis and toxicity of methanol extract of Etlingera elatior (torch ginger) flowers. Asian Pac J Trop Dis 3 (10): 769-774.

Lutfia A, Munir E, Yurnaliza, Basyuni M. 2019b. Screening of antagonistic fungi from Etlingera littoralis (J.König) Giseke rhizome in Sibolangit Forest, North Sumatra. J Phys Conf Ser 1351: 012025

Lutfia A, Munir E, Yurnaliza. 2019a. Antagonistic fungal endophytes colonizing rhizome of Amomum centrocephalum A.D. Poulsen from North Sumatera, Indonesia. Intl J Adv Sci Eng Inf Technol 9 (4) $1427-1433$

Lutfia A, Munir E, Yurnaliza. 2019c. Antagonistic endophytic fungi of Hedychium coronarium J. Koenig from Hutan Sibayak and Taman Hutan Raya, North Sumatra against Staphylococcus aureus ATCC® $29213^{\mathrm{TM}}$. IOP Conf Ser Earth Environ Sci 305: 012002. DOI $10.1088 / 1755-1315 / 305 / 1 / 012019$.

Lutfia A, Munir E, Yurnaliza. 2019d. Antagonistic endophytic fungi of Globba pendula Roxb. from Taman Hutan Raya, North Sumatra against Staphylococcus aureus ATCC® $29213^{\mathrm{TM}}$. IOP Conf Ser Earth Environ Sci 305: 012003. DOI: 10.1088/1755-1315/305/1/012003;

Manter DK, Vivanco, JM. 2007. Use of the ITS Primers, ITS1F and ITS4, to characterize fungal abundance and diversity in mixed-template samples by qPCR and length heterogeneity analysis. J Microbiol Methods 71 (1): 7-14.

Mukesh S, Vipul K, Shahid M, Sonika P, Singh A. 2016. Trichoderma - a potential and effective bio fungicide and alternative source against notable phytopathogens: a review. Afr J Agr Res 11 (5): 310-316.

Munir E, Lutfia A, Yurnaliza. 2019. Records of culturable endophytic fungi inhabiting rhizome of Elettaria in Hutan Sibayak, North Sumatera. IOP Conf Ser Earth Environ Sci 305: 012004. DOI 10.1088/1755-1315/305/1/012004.

Nei M, Kumar S. 2000. Molecular Evolution and Phylogenetics. Oxford University Press, New York.

Oliveira BR, Crespo MTB, Romao MVS, Benoliel MJ, Samson RA, Pereira VJ. 2013. New insights concerning the occurrence of fungi in water sources and their potential pathogenicity. Water Res 47 (16) 6338-6347.

Park Y, Mishra RC, Yoon S, Kim H, Park C, Seo S, Bae H. 2019 Endophytic Trichoderma citrinoviride isolated from mountaincultivated ginseng (Panax ginseng) has great potential as a biocontrol agent against ginseng pathogens. J Ginseng Res. 43 (3): 408-420.
Punnawich Y, Montree I, Warin I, Kan C. 2009. Antifungal effects of Thai medicinal plants against Colletotrichum gloeosporioides Penz. Philipp Agric Sci 92 (3): 265-270.

Sharma P. 2011. Complexity of Trichoderma-Fusarium interaction and manifestation of biological control. Aust J Crop Sci 5 (8): 1027-1038.

Shi X, Wang D, Li X, Li L, Meng L, Li X, Pi Y, Zhou X, Wang B. 2017. Antimicrobial polyketides from: Trichoderma koningiopsis QA-3, an endophytic fungus obtained from the medicinal plant Artemisia argyi. RSC Adv 81 (7): 51335-51342.

Silalahi M, Nisyawati. 2018. The ethnobotanical study of edible and medicinal plants in the home garden of Batak Karo sub-ethnic in North Sumatra, Indonesia. Biodiversitas 19 (1): 229-238.

Silalahi M, Supriatna J, Walujo EK, Nisyawati. 2015. Local knowledge of medicinal plants in sub-ethnic Batak Simalungun of North Sumatra, Indonesia. Biodiversitas 16 (1): 44-54.

Siregar, ES, Pasaribu N. 2008. Inventarisasi jenis-jenis Zingiberaceae di Hutan Sibayak Sumatera Utara. Jurnal Penelitian MIPA 2 (1): 22-24.

Strobel G, Daisy B. 2003. Bioprospecting for microbial endophytes and their natural products. Microbiol Mol Biol Rev 67 (4): 491-502.

Strobel G. 2003. Endophytes as sources of bioactive products. Microb Infect 5 (6): 535-544.

Sung WS, Lee DG. 2010. Antifungal action of chlorogenic acid against pathogenic fungi, mediated by membrane disruption. Pure Appl Chem 82 (1): 219-226

Suryanto D, Yeldi N, Munir E. 2016. Antifungal activity of endophyte bacterial isolates from torch ginger (Etlingera elicitor (Jack.) R. M Smith)) root to some pathogenic fungal isolates. Intl J Pharmtech Res 9 (8): 340-347.

Uzma F, Konappa NM, Chowdappa S. 2016. Diversity and extracellular enzyme activities of fungal endophytes isolated from medicinal plants of Western Ghats, Karnataka. Egypt J Basic App Sci 3 (4): 335-342.

Venieraki A, Dimou D, Katinakis P. 2017. Endophytic fungi residing in medicinal plants have the ability to produce the same or similar pharmacologically active secondary metabolites as their hosts. Hell Plant Prot J 10 (2): 51-66.

Vu D, Groenewald M, de Vries M, Gehrmann T, Stielow B, Eberhardt U, Al-Hatmi A, Groenewald JZ, Cardinali G, Houbraken J, Bouekhout T, Crous PW, Robert V, Verkley GJM. .2019. Large-scale generation and analysis of filamentous fungal DNA barcodes boosts coverage for kingdom fungi and reveals thresholds for fungal species and higher taxon delimitation. Stud Mycol 92: 135-154.

Wei JG, Xu T, Guo LD, Liu AR, Zhang Y, Pan XH. 2007. Endophytic Pestaliotopsis species associated with plants of Podocarpaceae, Theaceae and Taxaceae in Southern China. Fungal Divers 24: 55-74.

Yurnaliza Y, Aryantha INP, Esyanti RR, Susanto A. 2014. Antagonistic activity assessment of fungal endophytes from oil palm tissues against Ganoderma boninense Pat. Plant Pathol J 13 (4): 257-267. 